\title{
Characterization of micromachined silicon membranes for immunoisolation and bioseparation applications
}

\author{
Tejal A. Desai ${ }^{\mathrm{a},}$, Derek Hansford ${ }^{1, \mathrm{~b}}$, Mauro Ferrari ${ }^{2, \mathrm{c}}$

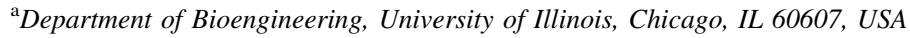 \\ ${ }^{\mathrm{b}}$ Biomedical Microdevices Center, University of California, Berkeley, CA 94720, USA \\ ${ }^{\mathrm{c}}$ Center for Bioengineering, The Ohio State University, Columbus, OH 43210, USA
}

Received 20 November 1998; received in revised form 4 February 1999; accepted 5 February 1999

\begin{abstract}
The application of microfabrication technology to create precise separation and isolation membranes for biomedical applications is described. By utilizing fabrication techniques commonly employed in the microelectronics industry (MEMS), membranes can be fabricated with well-controlled and uniform pore sizes, allowing the optimization of membrane parameters for biomedical applications in cell immunoisolation and viral filtration. Using bulk and surface micromachining to create diffusion membranes, pore sizes down to $18 \mathrm{~nm}$ have been attained through deposition and subsequent etching of sacrificial layers on silicon. Membranes were shown to be sufficiently permeable to small biomolecules such as oxygen, glucose, and insulin, while excluding the passage of larger proteins such as immunoglobulin $\mathrm{G}$ ( $\mathrm{IgG}$ ). The semipermeability of microfabricated membranes, their biocompatibility, ease in sterilization, along with their thermal and chemical stability, may provide a significant advantages for biomedical applications. Microfabrication technology may also be applied to other materials of interest for the development of highly controlled membranes. (C) 1999 Elsevier Science B.V. All rights reserved.
\end{abstract}

Keywords: Bioseparation; Biotechnology; Inorganic membranes; Immunoisolation; Membrane; Micromachining; Protein diffusion

\section{Introduction}

The immunoprotection of hormone-secreting cells and tissue with a semipermeable membrane prior to transplantation is a promising therapy for a wide variety of diseases including diabetes, Parkinson's,

\footnotetext{
*Corresponding author. Tel.: +1-312-413-8723; fax: +1-312996-5921; e-mail: tdesai@uic.edu

${ }^{1}$ Tel.: +1-510-643-7035; fax: +1-510-643-8928; e-mail: dhans@uclink2.berkeley.edu

${ }^{2}$ Tel.: +1-614-292-4756; fax: +1-614-292-7301; e-mail: ferrari.5@osu.edu
}

and hemophilia [1-3]. For the encapsulation of insulin-secreting pancreatic islets, it has been established that the encapsulating membrane must be sufficiently permeable to insulin and glucose, while at the same time, impermeable to immunologic cells, antibodies, and other immune molecules, which might lead to destruction of the transplanted cells [1].

Nonetheless, there is much experimental variability, both in vitro and in vivo, as to the exact transport properties required for immunoisolation membranes. These membranes have been poorly characterized in terms of their specific large and small molecule diffusion parameters, focusing instead on absolute mem- 
brane performance criteria [4]. Indeed, it is often complicated to characterize the diffusive transport properties of immunoisolation membranes due to the variability and asymmetry of these membranes, most of which are polymeric. This nonuniformity in membrane architecture makes it difficult to define diffusion lengths and absolute molecular weight cut-off dimensions of the membrane.

Typically, transport in polymeric immunoisolation membranes is characterized by the nominal molecular weight cut-off (MWCO) of the membrane [1]. However, polymeric biocapsules usually have a broad pore size distribution, and cannot provide an absolute size or molecular weight cut-off [3]. Furthermore, polymeric membrane thicknesses are not always uniform, and on the order of $100 \mu \mathrm{m}$. Although the nominal molecular weight cut-off gives us an idea as to which molecules can pass through a membrane, this parameter is not always useful in determining the rate of passage or absolute selectivity through diffusion based immunoisolation membranes. It is often difficult to define diffusion lengths, pore sizes, and distributions in polymeric membranes $[5,6]$.

\subsection{Current membrane technology}

Several different types of polymer membranes have been investigated for immunoisolation. However, despite years of research, there is no consensus as to the ideal pore size or molecular weight cut off for immunoisolation and protein separation. For example, Maki et al. [7] characterized acrylic co-polymer membranes that had a nominal molecular weight cut-off of approximately 50000-80000 Dalton which inhibited the passage of IgG, but did not completely block IgG passage. Agarose $(5 \%)$ immunoisolation membranes characterized by Iwata et al. [8], showed significant permeability to IgG and serum complement proteins, but still provided short term immunoisolation. Similarly, Burczak et al. [9] found that poly vinyl alcohol (PVA) membranes with number average molecular weights of either 48000 or 247000 also resulted in $\mathrm{IgG}$ passage. Such studies indicate the variations inherent in polymeric membrane pore size and the difficulties in preventing of the passage of immunoglobulins through such membranes.

Due to the instabilities of some polymeric membranes in vivo, changes in molecular weight cut-offs or pore size geometry are observed over time. Factors such as temperature and $\mathrm{pH}$ often drastically alter membrane properties. Such was the case for poly(acrylonitrile-co-vinyl chloride) (P(AN/VC)) membranes which had an initial molecular weight cutoff (at 90\% rejection) of approximately 40000 and then 54000 at twelve months after implantation [10].

Therefore, microfabricated inorganic membranes, with uniform and well-controlled pore sizes in the tens of nanometer range, may be able to provide better size-based immunoisolation than conventional polymeric biocapsule membranes. Using techniques such as thin-film deposition, photolithography, and etching that were originally developed for the microelectronics and integrated circuits industry, substrates with well-defined micro- and nano-architectures and features can be created. Due to the uniformity and known geometry of microfabricated membranes, it is much more straightforward to analyze and determine transport characteristics and diffusion coefficients [11]. Although microfabricated membranes, both polymeric and inorganic, have been proposed previously, these membranes had pores that were defined by standard photolithography, and were therefore limited to micron size pore diameters. Therefore, these membranes could not be applied to biomedical applications involving immunoisolation and bioseparation $[12,13]$. The microfabricated membranes presented here use novel processing strategies to achieve nanopores.

Moreover, these membranes may aid in our knowledge of basic mechanisms in cellular immunoisolation. By understanding the exclusion and diffusion parameters of a given immunoisolation membrane, optimization of passage of nutrients and hormonal product can be achieved as well as more accurate assessments of immunoprotective capabilities of the membrane. For instance, upon implantation of foreign cells, an immunogenic response immediately develops. This sequence of events begins with the binding of antibodies (either IgG or IgM) to both cell surface and soluble antigens. Its diffusion is often regarded as the rate-determining step of islet cell destruction [2]. This is especially critical with immunoisolation of xenogenic tissue. Nonetheless, few studies have investigated the permeability of immunoisolative devices to specific immune molecules such as IgG, other than in a qualitative fashion [14,15]. 
Therefore, membranes with a range of pore sizes $(18,66$ and $78 \mathrm{~nm})$ were fabricated using bulk and surface micromachining and their permeability to insulin, glucose, and IgG was studied. The semipermeability of microfabricated membranes, along with their stability and biocompatibility, may provide an improved approach to the immunoisolation of cell xenografts.

\section{Materials and methods}

\subsection{Membrane fabrication}

The membrane fabrication protocol uses a combination of surface micromachining to define the actual membrane pores and bulk micromachining to create larger anisotropically-etched recesses in a single-crystal silicon wafer (Fig. 1). The standard microfabrication techniques of diffusion, thin film deposition,

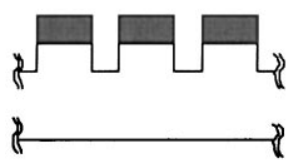

(a)

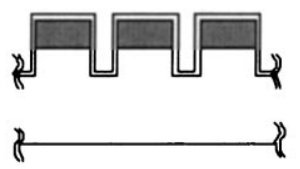

(c)

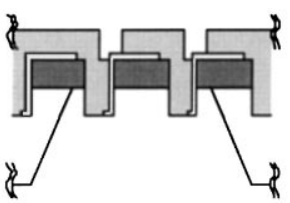

(b)

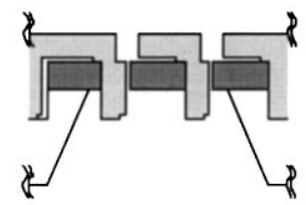

(d)

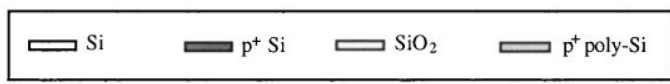

Fig. 1. Essential fabrication steps for the microfabricated biocapsule (not to scale): (a) A 4 in. (1 00 ) $\mathrm{Si}$ wafer is first B-doped $\left(>10^{20} \mathrm{~cm}^{-3}\right) .8 \mu \mathrm{m}$-deep exit holes are then etched through the $\mathrm{p}$ doped region. (b) A $20-100 \mathrm{~nm}$ sacrificial oxide layer, which defines the diffusion channel, is deposited using dry thermal oxidation. (c) Anchor points are opened in the oxide and a $3 \mu \mathrm{m}$ thick, B-doped $\left(>10^{20} \mathrm{~cm}^{-3}\right)$ polysilicon layer is deposited. A final etch step is performed to define entry holes in the polysilicon. (d) An EDP (ethylene diamine-pyrocatechol) etch is used to form the half-capsule. This will selectively etch the Si substrate without attacking the B-doped silicon and polysilicon layer, or the oxide. Following the etch, the channels are released with 5:1 buffered HF. photolithography, and etching are used in the creation of the membranes. The detailed fabrication strategy has been described in detail previously [11] and is schematically depicted in Fig. 1.

Briefly, exit holes are plasma-etched through a boron-doped silicon substrate (Fig. 1(a)). Next, in the most critical step, a sacrificial oxide is grown using dry thermal oxidation (Fig. 1(b)). The thickness of this layer, which can be controlled to $\pm 1 \mathrm{~nm}$ as verified by ellipsometry and profilometry, will determine the final channel pore size. Typical thicknesses are in the range $10-100 \mathrm{~nm}$. Following the oxidation, a $2 \mu \mathrm{m}$ borondoped polysilicon layer is deposited over the oxide. Another plasma etch is performed to produce the entry holes, the location of which is slightly offset from the position of the exit holes. The backside is then anisotropically etched in EDP solution to expose the backside of the membrane (Fig. 1(c)). Finally, the sacrificial oxide layer (deposited in step 1b) is removed by selectively etching it with concentrated HF (Fig. 1(d)). This generates the actual immunoisolating diffusion pores of the membrane. Following membrane processing and anisotropic etching, a dicing step is performed to separate each die and create individual membranes that can be characterized.

The membranes can be tested individually or two membranes can be bonded together using a polydimethyl siloxane bonding agent to produce a microfabricated biocapsule (Fig. 2). Briefly, the desired cells or biomolecules of interest are placed inside one half-capsule, and the encapsulation is completed by bonding to another half-capsule with a thin adhesive poly dimethyl siloxane layer that is patterned on the outer membrane edges [16]. The final membrane dimensions are as follows: $550 \mu \mathrm{m}$ in bulk thickness, $2 \times 2 \mathrm{~mm}$ in overall lateral dimensions, membrane area of $10.4 \mathrm{~mm}^{2}$, cavity volume of approximately $5 \mu \mathrm{l}$, and a membrane thickness of $9 \mu \mathrm{m}$. The number of pores per centimeter for this current design is $1.4 \times 10^{6}$ although it is possible to increase the pore density to $10^{7} \mathrm{~cm}^{-2}$. The actual diffusion pores have rectangular geometry with minimum channel dimension of 78,66 , or $18 \mathrm{~nm}$.

\subsection{Characterization}

The dimensions of the pores are determined by measuring the thickness of the deposited sacrificial 

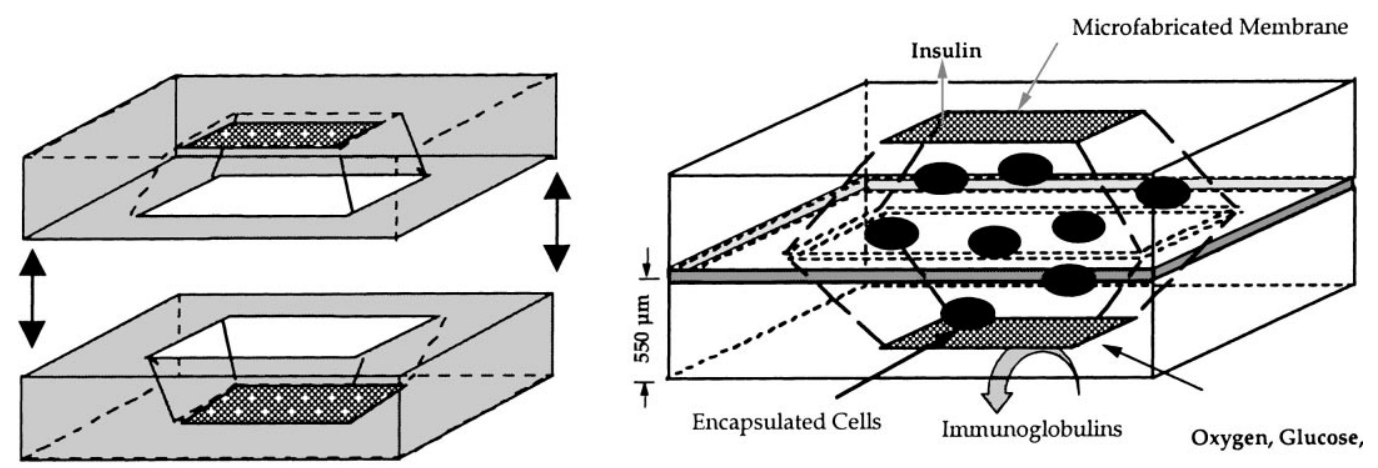

Fig. 2. Schematic of assembled biocapsule consisting of two micromachined membranes bonded together to form a cell-containing cavity bounded by membranes.

oxide layer by ellipsometry. The variations of thickness over a 4 in. wafer are less than $5 \%$. Such control in channel size cannot be achieved in conventional polymeric materials, such as cellulose acetate or polyvinylidene fluoride [2]. However, with this current membrane design, slightly larger than $5 \%$ of the surface area is used for diffusion. This can be increased depending on type of application. Membrane surfaces were also characterized optically via a reflected light microscope for macroscopic defect in membrane fabrication.

A low pressure bubble point test was used to characterize the uniformity of the pores and the presence of defects. The membrane is pre-wetted with deionized water and then immersed into the liquid. Pressure is applied from the back of the membrane to drive the liquid out of the membrane pores. At a certain pressure, the liquid is forced out of the pores and bubble will form at the pore exit. The bubble point pressure is inversely related to maximum pore size. Any region with microscopic holes or cracks produces differences in bubble formation, thus making it possible to identify microscopic defects. Membranes were also examined under scanning electron microscope from the top view and in cross-section (Fig. 3).

\subsection{Diffusion of polystyrene beads of various dimensions}

The ability of microfabricated biocapsule membranes to perform size-based exclusion of biomolecules was studied using FITC-labeled polystyrene beads of 44 and $100 \mathrm{~nm}$, the sizes that were commercially available. Fig. 4 schematically illustrates the size-based separation of beads and/or biomolecules through microfabricated membranes. A suspension of beads was placed into a half-biocapsule well $(2 \mu 1)$ and subsequently sealed with another biocapsule half. At intervals of $24 \mathrm{~h}$, the medium surrounding the biocapsules were collected and bead concentration was measured using a spectrofluorimeter.

\subsection{Diffusion of glucose and insulin through microfabricated membranes}

The ability of the membrane to allow diffusion of glucose and insulin while restricting the passage of IgG was studied in vitro by measuring relative concentrations of the desired molecule on both sides of the membrane using a mini-diffusion chamber and monitoring diffusion out of the actual biocapsule [9]. The mini-diffusion chamber was designed and machined out of transparent acrylic. It consisted of two compartments A and B with fixed volumes of $2 \mathrm{ml}$, separated by the biocapsule membrane, sealed with $o$-rings, and finally screwed together (Fig. 5). The diffusion chamber was rotated at a fixed speed of $10 \mathrm{rpm}$ to ensure adequate mixing of the solutions throughout the course of the diffusion experiments. The concentration of nutrient and immune molecules of interest was measured on either side of the membrane to determine membrane permeability.

At time zero, glucose or insulin was added to compartment $\mathrm{A}$ and $1 \mathrm{ml}$ aliquots of medium 


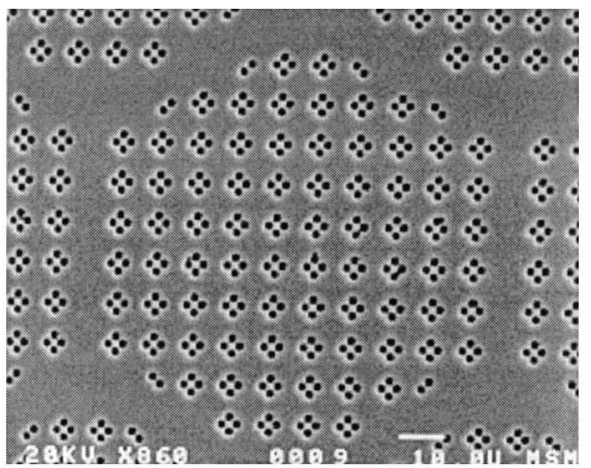

(a)

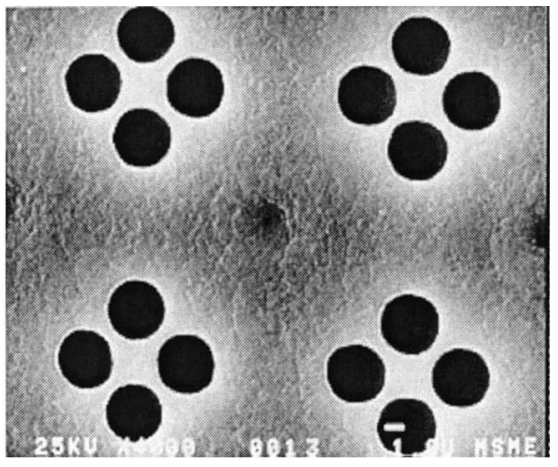

(c)

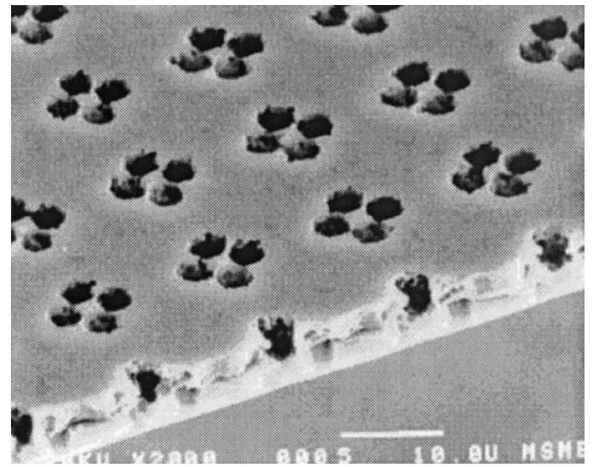

(b)

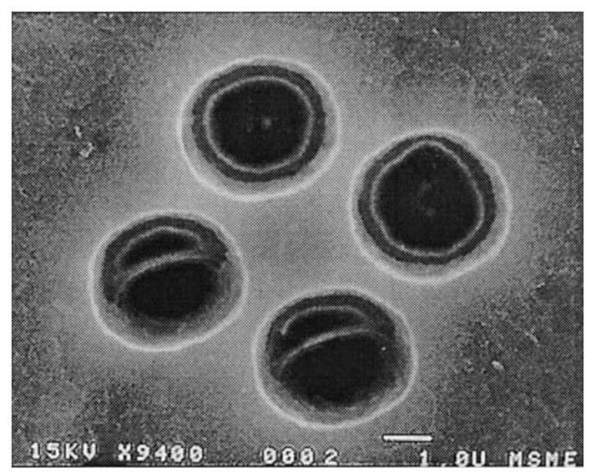

(d)

Fig. 3. Scanning electron micrograph of the microfabricated membrane of $18 \mathrm{~nm}$ pore size: (a) top-view of pore array; (b) cross sectional view of membrane; (c) cluster of $2 \times 2$ micron entry ports; (d) magnified view of $2 \times 2$ micron entry ports with 18 nm channels underneath.

from compartment B were taken at various time points. Glucose concentrations were determined by the method of Huggett and Nixon [17] and insulin concentration was measured by radioimmunoassay.

Islet cells were then cultured on silicon membranes of pore sizes of $18 \mathrm{~nm}, 78 \mathrm{~nm}$, and 3 microns. Approximately 30 islets were placed in each sample (volume of approximately $5 \mu \mathrm{l}$ ). Glucose-supplemented medium was allowed to diffuse to the islets, from underneath the membrane, to stimulate insulin production. Similarly, groups of thirty unencapsulated islets were cultured in 24 well culture dishes and stimulated and stimulated with glucose-supplemented medium. The concentration of insulin, secreted by the islets through the membrane, into the surrounding medium was compared between the unencapsulated islets and the islets on micromachined membranes.
The insulin release by encapsulated islets was evaluated by adding stimulatory medium to wells containing islet-filled biocapsules. Immediately after adding the solutions (time zero), aliquots of medium were collected from each of the wells. Samples were collected at $10 \mathrm{~min}$ intervals, while minimizing the sampling time during which biocapsules were out of the incubator. It was important to control experimental temperature, since insulin secretion in response to glucose is reduced at room temperature and molecular diffusion would be also reduced at lower temperatures. Insulin secretion over $90 \mathrm{~min}$ was calculated by taking into account the final incubation volume and the insulin removed in the four successive samplings [5]. The collected samples were stored at $-20^{\circ} \mathrm{C}$ until radioimmunoassayed, as described above. Mean values \pm SD of the amount of insulin were calculated for each group at each time point. 

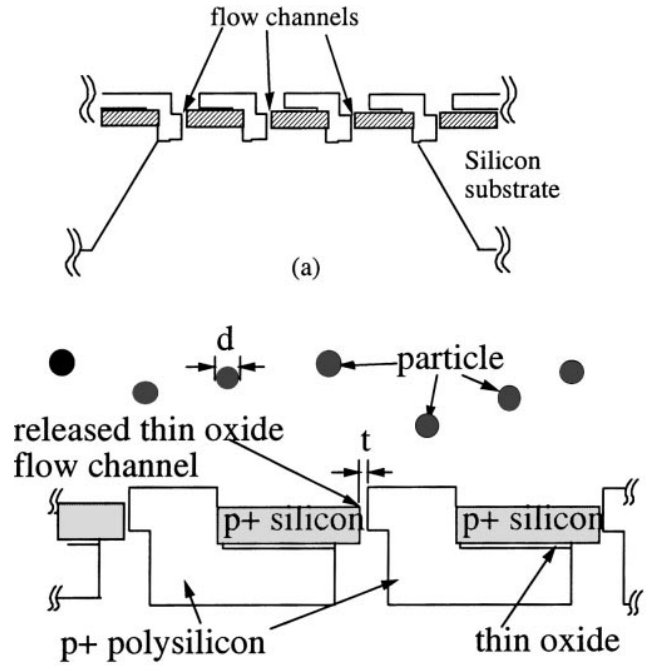

(b)

Fig. 4. Schematic of (a) microfabricated multifunctional membrane design and (b) permselectivity based on size, shape, and/or charge.

\subsection{IgG diffusion through microfabricated membranes}

Permeability of $\operatorname{IgG}$ through the microfabricated biocapsule membrane was examined using FITClabeled IgG. This marker was placed on one side of the diffusion chamber and diffusion through the membrane into the other chamber was monitored. Membranes of 18,66 , and $78 \mathrm{~nm}$ pore size were tested. The diffused FITC-IgG was detected by using a spectrofluorimeter (excitation wavelength $495 \mathrm{~nm}$, emission

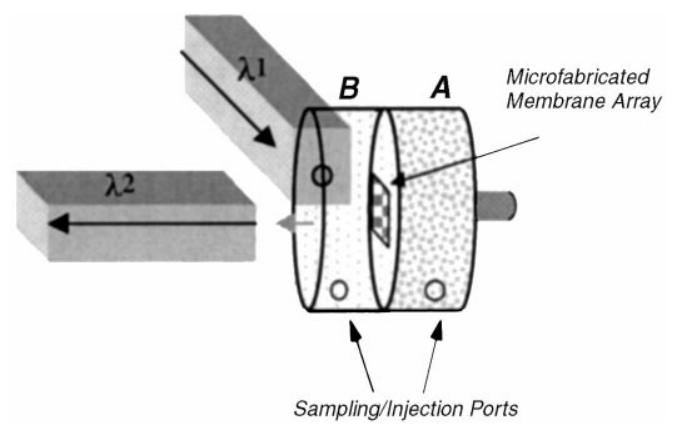

Fig. 5. Rotating diffusion chamber used to test microfabricated membranes. wavelength $525 \mathrm{~nm}$ ). The concentration of $\mathrm{IgG}$ was monitored daily for four days.

Additionally, IgG diffusion was measured directly from full biocapsules. The molecule of interest was pipetted into one half biocapsule wells and two half capsule were bonded together to form a full biocapsule [16]. Biocapsules with two membrane pore sizes were examined: 18 and $66 \mathrm{~nm}$. At time zero, FITC labeled IgG was added to each biocapsule well $(2 \mu \mathrm{l})$ and sealed. At various time intervals, an aliquot $(1 \mathrm{ml})$ of the medium surrounding each biocapsule was taken. Diffusion of IgG out of fully assembled biocapsule into surrounding culture medium was monitored. At various time intervals, an aliquot of the medium surrounding each biocapsule was taken. FITC labeled IgG concentrations were also determined using a spectrofluorimeter (excitation wavelength $495 \mathrm{~nm}$, emission wavelength $525 \mathrm{~nm}$ ) and diffusion was monitored over a period of four days [14].

\subsection{Evaluation of cellular immunoprotective properties}

To determine whether microfabricated membranes could provide cellular immunoprotection specifically to pancreatic islets, the functionality of both encapsulated and unencapsulated islets in presence of serum complement and antibody was studied using a tissue culture method [15].

\subsubsection{Isolation of pancreatic islets of Langerhans}

Pancreatic islets cells were isolated from neonatal rats by standard methods using a modified version of the protocol by Hellerstrom et al. [18] as described previously by Desai et al. [19]. Briefly, the steps include removal of pancreatic tissue, mechanical fragmentation of tissue, enzymatic digestion using collagenase, and sedimentation to separate islets. Islets were cultured several days before use.

The survival of pancreatic islets was used to monitor immunoprotective properties of islet filled biocapsules. Biocapsules with membranes of 78, 66, and $18 \mathrm{~nm}$ pore sizes were studied. For experiments, membranes were sterilized and placed into sterile tissue culture containers. Microfabricated membranes were filled with a suspension of islets and bonded together as described in Desai et al. [19]. Free islets and islet filled biocapsules (10 islets per capsule) 
were incubated in cell culture medium (RPMI) with anti-rat rabbit serum and rabbit complement at $37^{\circ} \mathrm{C}$ (Sigma, St. Louis, MO). Unencapsulated islets were incubated under the same conditions. Islet viability and functionality was determined by measuring insulin concentrations in the supernatant fluid after stationary glucose stimulations $(16.7 \mathrm{mM})$. The presence of insulin in solution was determined using a radioimmunoassay and detection by a gamma counter. The ability of the islets in various pore-sized biocapsules to maintain responsiveness to glucose stimulation in the presence of anti-rat islet antibodies and complement was compared to that of unencapsulated islets.

\section{Data and results}

\subsection{Diffusion of polystyrene beads of various dimensions}

It was found that biocapsules membranes of $18 \mathrm{~nm}$ pore size completely blocked the diffusion of 44 and $100 \mathrm{~nm}$ diameter polystyrene beads, while $66 \mathrm{~nm}$ pore sized membranes only blocked $100 \mathrm{~nm}$ diameter beads. No fluorescent signal above baseline was detected in the incubation medium surrounding $18 \mathrm{~nm}$ biocapsules after 1 and 4 days. This suggests that the biocapsule achieved absolute retention of the beads.

\subsection{Glucose and insulin diffusion}

The concentration of insulin, secreted by the islets through the membrane, into the surrounding medium was compared between the unencapsulated islets and the islets on micromachined membranes. The concentration of diffused insulin through the membrane into the medium was compared to the amount of insulin secreted by unencapsulated islets. The amounts were similar in concentration and time release suggesting that glucose was able to sufficiently pass through the pores of the wafer pockets to stimulate islets for insulin production.

Results indicated that the insulin secretion by the islets and subsequent diffusion through the biocapsule membrane channels was similar to that of unencapsulated islets for both 3 micron and $78 \mathrm{~nm}$ pore sized membranes, with insulin diffusion though the membrane occurring within 10 min of stimulation. Figs. 6 and 7 show the typical insulin release profile in response to stimulatory $(16.7 \mathrm{mM})$ glucose medium over $1 \mathrm{~h}$ under static incubation for 3 micron, 78 and $18 \mathrm{~nm}$ pore-sized membranes. This profile indicated that insulin and glucose diffusion occurred at sufficiently high rates through the microfabricated mem-

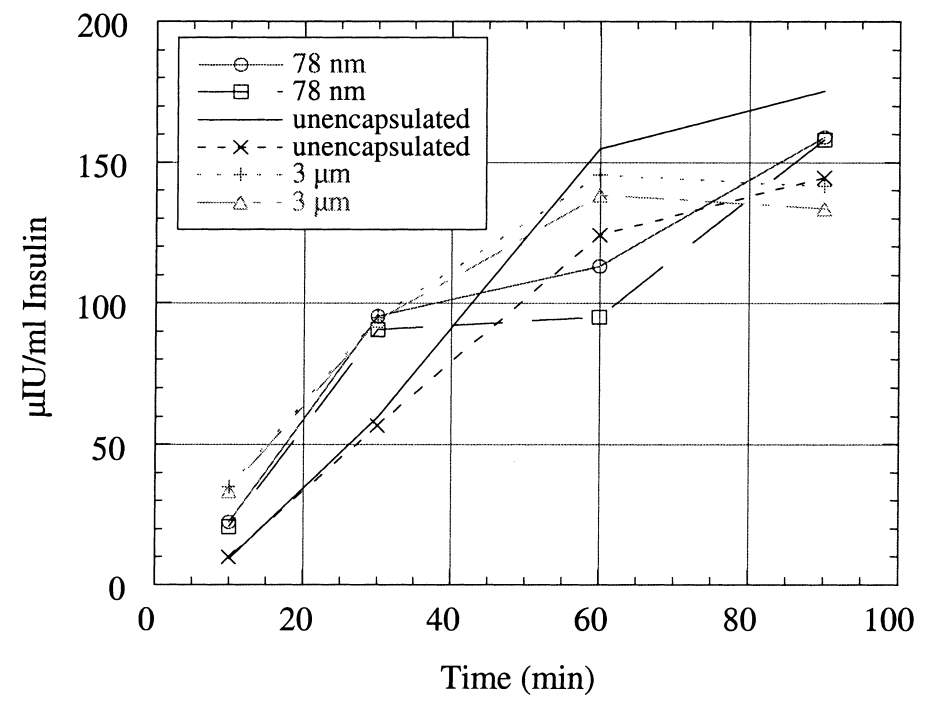

Fig. 6. Insulin diffusion through microfabricated membranes of differing pore size. 


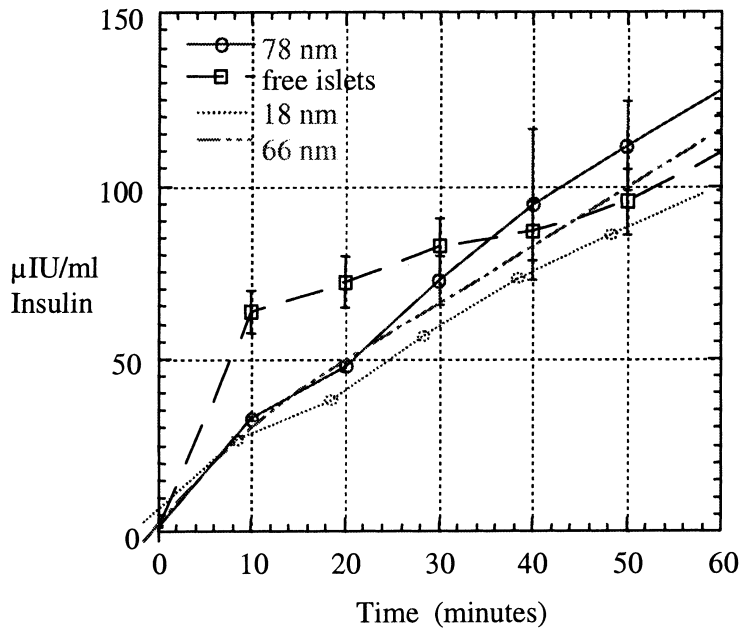

Fig. 7. Insulin diffusion over time for islet-filled biocapsule subjected to glucose stimulus at $t=0$.

brane to ensure nutrient exchange for encapsulated islet cells. These experiments show that no diffusion barrier is formed by the membrane for glucose and insulin, while taking into account the effect of rotation on mass transfer.

\subsection{IgG exclusion}

The data indicated that microfabricated biocapsule membranes retarded significant IgG passage but did not completely prevent IgG penetration. As Fig. 8 shows, although membranes with $18 \mathrm{~nm}$ pore size had a great immunoisolatory effect, both 66 and $78 \mathrm{~nm}$ pore-sized membranes allowed significant quantities of $\mathrm{IgG}$ through over time. The percent of IgG diffusion (concentration of $\mathrm{IgG}$ that passes through the membrane) was less than $0.4 \%$ after $24 \mathrm{~h}$ and $2 \%$ after over $150 \mathrm{~h}$ through the $18 \mathrm{~nm}$ membranes (Fig. 9). Compared to commonly used polymeric membranes, this rate was several times smaller indicating superior immunoprotection. For example, Dionne et al. [14] measured an IgG concentration of $1 \%$ after $24 \mathrm{~h}$ through poly(acrylonitrileco-vinyl chloride) membranes with a molecular weight cut-off of $\approx 80000 \mathrm{MW}$.

Diffusion across the membrane can be described by,

$N=\frac{A_{\mathrm{eff}} D_{\mathrm{eff}}}{L}\left(C_{\mathrm{A}}-C_{\mathrm{B}}\right)$

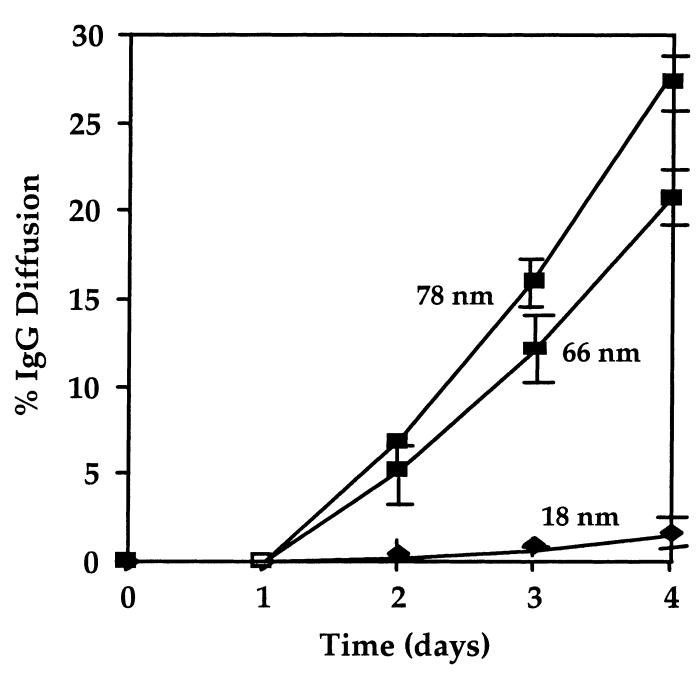

Fig. 8. IgG diffusion through microfabricated biocapsules of three different pore sizes.

where $N$ is the flux, $A_{\text {eff }}$ the cross sectional pore area, $D$ the effective diffusivity, $L$ the membrane thickness, and $C$ is the chamber concentration $[9,14]$. It is assumed that (i) there were no significant boundary layer effects in the rotating diffusion chamber and (ii) a steady-state concentration profile was established within the membrane. By coupling this with the massbalance equation,

$N=-\frac{\mathrm{d}\left(V_{\mathrm{A}} C_{\mathrm{A}}\right)}{\mathrm{d} t}=\frac{\mathrm{d}\left(V_{\mathrm{B}} C_{\mathrm{B}}\right)}{\mathrm{d} t}$

one can find the following relationship between con-

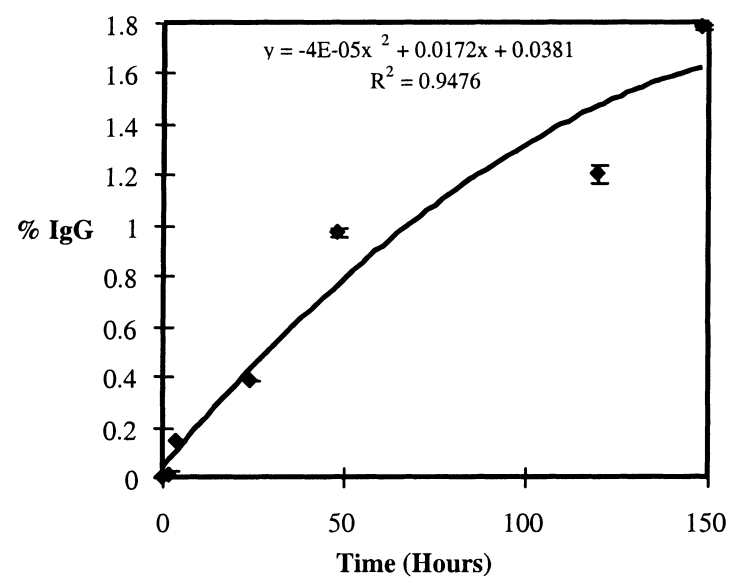

Fig. 9. Diffusion of $\mathrm{IgG}$ through $18 \mathrm{~nm}$ pore-sized membrane. 
Table 1

Membrane diffusion parameters for $\operatorname{IgG}$

\begin{tabular}{lll}
\hline Pore size $(\mathrm{nm})$ & Effective area $\left(\mathrm{cm}^{2}\right)$ & $D_{\text {eff }}$ \\
\hline 18 & 0.00003072 & $6.75 \mathrm{E}-12$ \\
66 & 0.00011264 & $2.25 \mathrm{E}-08$ \\
78 & 0.00013312 & $3.375 \mathrm{E}-08$ \\
\hline
\end{tabular}

centration in chamber B and time,

$\ln \frac{\alpha C_{\mathrm{A} 0}+C_{\mathrm{B} 0}-C_{\mathrm{B}}(1+\alpha)}{\alpha\left(C_{\mathrm{A} 0}-C_{\mathrm{B} 0}\right)}=-\left[\frac{1+\alpha}{\alpha}\right]\left[\frac{A_{\mathrm{eff}} D_{\mathrm{eff}}}{L V_{\mathrm{B}}}\right] t$

where $\alpha=$ volume fraction $=1, C_{\mathrm{A} 0}$ and $C_{\mathrm{B} 0}$ are initial solute concentrations in chamber $\mathrm{A}$ and $\mathrm{B}$, respectively, and $V_{\mathrm{B}}$ is the chamber volume $(=2 \mathrm{ml})$. By plotting,

$\ln \frac{\alpha C_{\mathrm{A} 0}+C_{\mathrm{B} 0}-C_{\mathrm{B}}(1+\alpha)}{\alpha\left(C_{\mathrm{A} 0}-C_{\mathrm{B} 0}\right)}$ vs. $t$

and since

$D_{\text {eff }}=-\frac{(\text { slope })\left(\alpha L V_{\mathrm{B}}\right)}{(1+\alpha) A_{\text {eff }}}$

the effective diffusivities of IgG for different pore-sized membranes can be determined (Table 1).

\subsection{Islet immunoprotection}

As shown in Fig. 9, the $18 \mathrm{~nm}$ biocapsules seem to provide significant immunoprotection to those islets

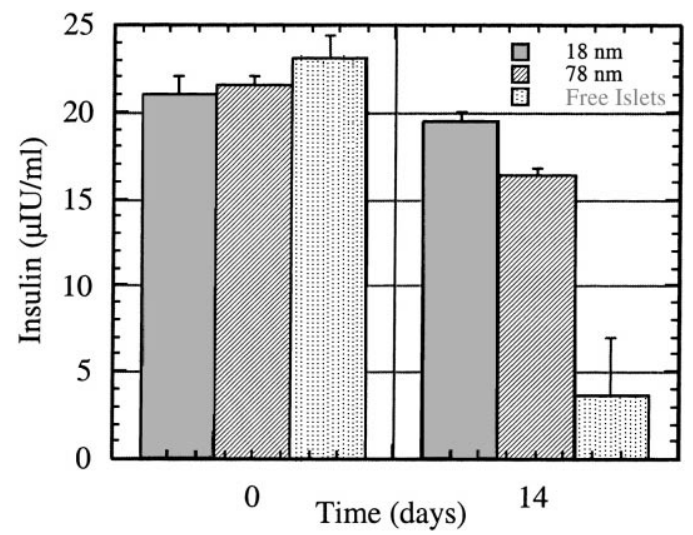

Fig. 10. Insulin secretion of islets within different pore-sized biocapsules and unencapsulated incubated for two weeks with serum complement/antibody solution (20 islets/biocapsule, $n=6$ ). encapsulated within its semipermeable membrane. After two weeks in culture with antibodies and serum complement, islets in microfabricated biocapsules maintained close to original glucose stimulated insulin secretive capability even after two weeks in the presence of antibodies and complement (see Fig. 10). Islets immunoprotected by $18 \mathrm{~nm}$ pore-sized membrane maintained their functionality better than those in $78 \mathrm{~nm}$ pore sized biocapsules, confirming that greater immunoprotectiveness was offered by $18 \mathrm{~nm}$ membranes. In contrast, there was a marked decrease in baseline and stimulated response in free islets. This suggests that microfabricated biocapsules with pore sizes in the $18 \mathrm{~nm}$ range may be used to immunoprotect transplanted cells.

\section{Discussion}

This study suggests that microfabricated biocapsules with membrane pore sizes less than $20 \mathrm{~nm}$, should be used to immunoprotect transplanted cells. It is realized that sufficient diffusion of insulin and simultaneous blockage of IgG is not a trivial parameter in the design of a successful biocapsule membrane. Although the IgG molecule has a molecular weight of approximately $150 \mathrm{kDa}$, studies have disagreed on the actual dimensions of the molecule, estimated to be tens of nanometers or less [20,21]. For example, Wang et al. [22] investigated permeability of relevant immune molecules to sodium alginate/poly-L-lysine capsules and found that significant amounts of IgG (close to 40\%) passed through both 230 and $110 \mathrm{kDa}$ membranes in $24 \mathrm{~h}$.

Our experiments showed that the passage of $\operatorname{IgG}$ through microfabricated membranes is greatly hindered, although not absolutely blocked. However, there are several possibilities why passage of antibodies through nanometer pore-sized membranes occurs. First, IgG diffusion may be due to small defects present in the membrane from fabrication processing. While examination of the membranes post processing showed no indications of macroscopic defects, there may have been submicron defects present in some of the membranes. The control and elimination of these submicron defects must be investigated to further improve immunoisolative properties of the biocapsule membrane. Furthermore, the bond- 
ing of the two membranes to form a biocapsule may not have provided a complete seal, and therefore, was prone to leakage of small immune molecules for the full capsule diffusion studies. Investigations into alternate bonding strategies are currently underway.

More importantly, the size, flexibility, and configuration of the $\mathrm{IgG}$ antibody may allow changes in conformation which permit its slow diffusion through pores greater than $18 \mathrm{~nm}$, despite its molecular diameter. Therefore, changing the pore geometry of the biocapsule may be able to provide a better barrier to $\mathrm{IgG}$ diffusion. Since the $\mathrm{IgG}$ molecule is not spherical, it is likely that membrane pore sizes as small as $10 \mathrm{~nm}$ may not completely block its passage [23]. However, using pore sizes in such small dimensions significantly decreases glucose and insulin diffusion as well. Therefore, it may be more appropriate to use membranes with pore sizes that can maintain the immunoisolation effect long-term in vivo, rather than guarantee absolute immunoisolation. For instance, alginate microcapsules that are $\mathrm{IgG}$ permeable and have membrane molecular weight cut-offs in excess of $70 \mathrm{kDa}$ (approximately $20 \mathrm{~nm}$ ) have been shown to protect islet xenografts $[1,24]$ at least short-term.

\section{Conclusion}

The microfabricated biocapsule has recently been introduced as an alternative to conventional polymeric immunoisolating biocapsules [19]. This study suggests that microfabricated biocapsules may be used to immunoprotect transplanted pancreatic islets by hindering the passage of IgG molecules while permitting the passage of glucose and insulin.

The microfabricated biocapsule represents an exciting alternative for immunoisolation of transplanted cells. With the advantages that microfabrication can offer in terms of achieving uniform and well-controlled pore diameter, length, and configuration, biocapsules can potentially be engineered and optimized precisely to one's desired specifications. This is especially important in developing a size-based immunoisolation device for transplanted cells such as pancreatic islets of Langerhans. Current studies are underway to investigate optimization of pore size to attain both immunoisolation and adequate hormonal diffusion out of the biocapsule for therapeutic appli- cations. Furthermore, biocapsules fabrication strategies are being modified to improve performance and reproducibility.

\section{References}

[1] R.P. Lanza, W. Chick, Encapsulated cell therapy, Scientific Am. Sci. Med. 2(4) (1995) 16-25.

[2] C.K. Colton, Implantable hybrid artificial organs, Cell Transplantation 4(4) (1995) 415-436.

[3] F. Lim, A.M. Sun, Microencapsulated islets as bioartificial endocrine pancreas, Science 210 (1980) 908-910.

[4] N.F. Sheppard, D.J. Mears, S.W. Straka, Micromachined silicon structures for modelling polymer matrix controlled release systems, J. Controlled Release 42 (1996) 15-24.

[5] L. Kessler, M. Pinget, M. Aprahamian, P. Dejardin, C. Damge, In vitro and in vivo studies of the properties of an artificial membrane for pancreatic islet encapsulation, Horm. Metab. Res. 23 (1991) 312-317.

[6] D. Chicheportiche, G. Reach, In vitro kinetics of insulin release by microencapsulated rat islets: effect of size of the microcapsules, Diabetologia 31 (1988) 54-57.

[7] T. Maki, J.J. O’Neil, J. Porter, C.J. Mullon, B.A. Solomon, A.P. Monaco, Porcine islets for xenotransplantation, Transplantation 62(1) (1996) 136-138.

[8] H. Iwata, K. Kobayashi, T. Takagi, T. Oka, H. Yang, H. Amemiya, T. Tsuji, T. Ito, Feasibility of agarose microbeads with xenogeneic islets as a bioartificial pancreas, J. Biomed. Mater Res. 28(9) (1994) 1003-1011.

[9] K. Burczak, T. Fujisato, M. Hatada, Y. Ikada, Protein permeation through poly(vinyl alcohol) hydrogel membranes, Biomaterials 15(3) (1994) 231-238.

[10] M.S. Shoichet, D.H. Rein, In vivo biostability of a polymeric hollow fibre membrane for cell encapsulation, Biomaterials 17(3) (1996) 285-290.

[11] W. Chu, M. Ferrari, Silicon nanofilter with absolute pore size and high mechanical strength, Microrobotics and Micromechanical Systems, SPIE Proc. 2593 (1995).

[12] C.J.M. van Rijn, C. Elwenspoek, IEEE Proceedings in Micro Electro Mechanical Systems, 1995, pp. 83-87.

[13] S.Y. Chou, P.R. Krauss, P.J. Renstrom, Science 272 (1996) 85 and 86.

[14] K.E. Dionne, B.M. Cain, R.H. Li, W.J. Bell, E.H. Doherty, D.H. Rein, M.J. Lysaught, F.T. Gentile, Transport characterization of membranes for immunoisolation, Biomaterials 17(3) (1996) 257-266.

[15] H. Iwata, N. Morikawa, T. Fujii, T. Takagi, T. Samejima, Y. Ikada, Does immunoisolation need to prevent the passage of antibodies and complement?, Transpl. Proc. 27(6) (1995) 3224-3226.

[16] T.A. Desai, W.H. Chu, J. Tu, P. Shrewbury, M. Ferrari, in: P.L. Gourley (Ed.), Microfabricated Biocapsules for Cell Xenografts: A Review, Micro and Nanofabricated Electro-OpticalMechanical Systems for Biomedical and Environmental Applications, SPIE 2978 (May 1997), pp. 216-226. 
[17] A.S. Huggett, D.A. Nixon, Use of a glucose oxydase, peroxydase and O-dianiside in determination of blood and urinary glucose, Lancet 2 (1957) 368-370.

[18] N.J. Hellerstrom, H. Lewis, R. Borg, N. Johnson, Freunkel, Method for large-scale isolation of pancreatic islets by tissue culture of fetal rat pancreas, Diabetes 28 (1979) 766-769.

[19] T.A. Desai, W.H. Chu, J.K. Tu, G.M. Beattie, A. Hayek, M. Ferrari, Microfabricated immunoisolating biocapsules, Biotechnol. Bioeng. 57 (1998) 118-120.

[20] P.C. Zhang, C. Bai, P.K. Ho, Y. Dai, Y.S. Wu, Observing Interactions Between the IgG Antigen and Anti-IgG Antibody with AFM, IEEE Engineering in Medicine and Biology, March/April 1997.
[21] C.K. Colton, E. Avgoustiniatos, Bioengineering in development of the hybrid artificial pancreas, Trans. ASME 113 (1991) 152-170.

[22] T. Wang, I. Lacik, M. Brissova, A. Anilkumar, A. Prokop, D. Hundeler, R. Green, K. Shahrokhi, A. Powers, An encapsulation system for the immunoisolation of pancreatic islets, Nature Biotechnol. 15 (1997) 358-362.

[23] H. Nakamura, in: T. Yamakawa (Ed.), Seikagaku Databook II. Tokyo, Japan, Tokyo Kagaku Dojin, 1981, p. 1107.

[24] W. Kuhtreiber, R.P. Lanza, W.L. Chick, Secretory function of biohybrid pancreas devices containing porcine islets, Asaio J. 40(3) (1994) M789-792. 